\title{
ON THE MOBILITY OF IONS IN AIR AT HIGH PRESSURES.
}

By A. J. Dempster.

A LTHOUGH several theories bearing on the mobility of ions in A gases have been given, none as yet can be said to be completely satisfactory. On this account it was thought that a determination of the mobility of ions in air at pressures much above those hitherto used might be of interest and perhaps afford further evidence on which to base theoretical considerations. The following paper contains an account of a series of such measurements.

\section{Mobility Measurements.}

In determining the mobilities the method used was practically that devised by Frank and Pohl. ${ }^{1}$ The air was ionized in the chamber $P G$, Fig. I, by polonium, which was placed on the plate $P$ facing the gauze $G$. As the pressures used were high the alpha rays from the polonium were all absorbed within the space $P G$. The plate $P$ was kept at a constant potential with regard to $G$, and $G$ was made alternately positive and negative by means of a battery of small storage cells and a commutator. At high pressures a difference of potential of 300 to 500 volts was required between $P$ and $G$ to get enough ions into the space $G S$ to give measurable deflections. The commutator was run at a constant rate and the charges received on the electrode $E$, for different voltages on $G$, were measured with a quadrant electrometer. The voltage between $G$ and $S$ when the ions were travelling towards $E$, which was always less than when they were travelling in the opposite direction, was measured with a Kelvin electrostatic voltmeter. Readings were taken with different voltages applied between $G$ and $S$ and from a curve representing these the voltage was determined which was just required for the ions to reach $E$ in the time interval in which they were moving. In some of the measurements two or three seconds elapsed between alternations of the potential of $G$. Under these conditions oscillations of the electrometer needle were set up by the induced charges, and so a second gauze $S$ was introduced and by means of a battery of small dry cells in the cylinder kept at a constant potential of 17 volts above zero. This device, which had been used by Lattey ${ }^{2}$ with success in his experiments almost entirely eliminated the swing of the needle due to induction.

1 Verh. der. Deutsch. Phys. Ges., p. 69, 1907.

2 Proc. Roy. Soc., July 28, 19 ro. 
[VoL. XXXIV.

The gauzes were supported by ebonite posts and the whole apparatus as described was placed in a steel cylinder which was filled with air to the

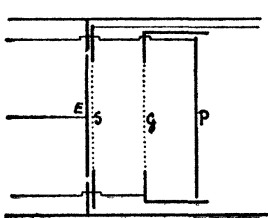

Fig. 1. pressure desired by means of the compressor of a liquid air apparatus. The air used was drawn through lime into the compressor and by it forced into a high pressure cylinder where it was of necessity in contact with water. From this cylinder it was passed through a second cylinder containing caustic potash in order to be dried and thence into the ionization chamber. The pressures were read on ordinary commercial pressure gauges which were afterwards calibrated with a standard gauge.

The commutator was made to run as uniformly as possible by keeping constant the electromotive force given by a small dynamo, with permanent magnets, connected with the commutator. The electromotive force of this dynamo was opposed by a couple of standard cells and the resultant difference of potential was measured with a second electrometer. With this arrangement slight variations could be readily detected and corrected.

The distance that the ions actually travelled could not be determined accurately by direct measurement. The distance between the two gauze sheets was $1.55 \mathrm{~cm}$. but as the meshes were considerable, being $1.65 \mathrm{~mm}$. in width, the path of the ions could only be said to be approximately I.55 $\mathrm{mm}$.

In the course of the experiments the velocity of the positive ion was found to vary inversely as the pressure, and so the value of the velocity found by other methods was assumed and the distance the ions travelled calculated to be $1.66 \mathrm{~cm}$. This distance was used therefore in calculating the mobility of the negative ion.

The following results were obtained where

$P_{1}=$ pressure in atmospheres $(\mathrm{I}$ atmo. $=76 \mathrm{cms}$. of mercury),

$k=$ mobility of the ion in $\mathrm{cm}$. per sec. per volt per $\mathrm{cm}$.,

$p k=$ product of the mobility and the pressure.

The measurements cited in Table I. were made one after another in the order given and the two principal sources of irregularity in obtaining them arose in making the pressure readings and in determining the critical voltage from the curves which represented the readings obtained with the different fields applied between $G$ and $S$. This uncertainty was greater in some measurements than in others and would largely account for the variations, shown in the table, of the product $p k$. These variations, however, are not large, and we may conclude therefore that the mobility of the positive ion varies inversely with the pressure up to as high as one hundred atmospheres. 
TABLE I.

Mobility of the Positive Ion in Air.

\begin{tabular}{c|c|c|c}
\hline \multicolumn{2}{c|}{ Pressure. } & $k$ & $p k$ \\
\hline Cm. of Hg. & Atmos. & $k$ & \\
\hline $7,726.16$ & 101.66 & .0139 & 1.42 \\
$7,726.16$ & 101.66 & .0138 & 1.41 \\
$6,410.80$ & 83.30 & .0154 & 1.28 \\
$4,496.16$ & 59.16 & .0227 & 1.34 \\
$2,971.60$ & 39.10 & .0344 & 1.35 \\
$1,989.68$ & 26.18 & .0517 & 1.36 \\
$1,086.30$ & 14.29 & .0945 & 1.35 \\
576.23 & 7.58 & .178 & 1.35 \\
379.85 & 5.00 & .262 & 1.31 \\
\hline
\end{tabular}

A summary of the measurements made on the mobility of the negative ion in air is given in Table II.

The first nine readings were taken before the measurements with the positive ion were made and the last five afterwards. The lower value obtained for the product $p k$ corresponding to the last five pressures cited can very probably be attributed to a change in the state of the air sup-

TABLE II.

Mobility of the Negative Ion in Air.

\begin{tabular}{c|r|r|l}
\hline \multicolumn{2}{c|}{ Pressure. } & \multicolumn{2}{c|}{} \\
\cline { 1 - 2 } Cm. of Hg. & Atmos. & $k$ & $p k$ \\
\cline { 1 - 2 } $7,922.24$ & 104.24 & .0224 & 2.33 \\
$6,304.96$ & 82.96 & .0281 & 2.33 \\
$4,547.84$ & 59.84 & .0378 & 2.26 \\
$3,152.48$ & 41.48 & .0532 & 2.21 \\
$3,152.48$ & 41.48 & .0536 & 2.22 \\
$2,945.76$ & 38.76 & .0561 & 2.18 \\
$2,585.16$ & 33.66 & .0637 & 2.15 \\
$1,757.12$ & 23.12 & .0906 & 2.10 \\
$1,086.04$ & 14.29 & .143 & 2.04 \\
$2,264.80$ & 29.80 & .064 & 1.96 \\
$1,550.40$ & 20.40 & .0915 & 1.87 \\
$1,033.60$ & 13.60 & .130 & 1.76 \\
558.14 & 7.34 & .253 & 1.86 \\
379.85 & 5.00 & .330 & 1.65 \\
\hline
\end{tabular}

plied by the liquid air machine, as the mobility of the negative ion in air at atmospheric pressure is known from Lattey's investigation to be greatly affected by changes in the amount of moisture present.

It is evident, however, from a survey of all the results given in Table II., that there is a gradual increase in the value of $p k$ as the pressure rises, since the ratio of the mobility of the negative ion to that of the positive 
[VoL. XXXIV.

ion at high pressures is about $\mathbf{1 . 7}$, while at the lowest pressures investigated this ratio was only about $\mathbf{I . 2 6}$.

\section{Mobility and Potential Difference in Antechamber.}

The above readings were all made with a potential difference of more than 300 volts between $P$ and $G$. At low pressures measurable deflections could also be obtained with a much lower potential difference than this voltage, but it was noticed that the velocity appeared to depend on the strength of the field in which the ions were made, for example, at a pressure of 7.59 atmospheres the positive ion made in a field of 319 volts between $P$ and $G$ had an apparent velocity 1.47 times the velocity when made in a field of ro volts between $P$ and $G$. The following groups of measurements which illustrate this point were made with different potentials $V$ between $P$ and $G$, each group being made with the same sample of air.

\begin{tabular}{|c|c|c|c|c|}
\hline \multicolumn{2}{|c|}{ Pressure. } & \multirow{2}{*}{ V } & \multirow{2}{*}{$k$} & \multirow{2}{*}{$p k$} \\
\hline $\mathrm{Cm}$. of $\mathrm{Hg}$. & Atmos. & & & \\
\hline \multirow[t]{2}{*}{379.85} & 5.00 & 384 & .262 & 1.31 \\
\hline & & 10 & .186 & .95 \\
\hline \multirow[t]{5}{*}{576.23} & 7.59 & 319 & .178 & 1.35 \\
\hline & & 83 & .167 & 1.27 \\
\hline & & 39 & .155 & 1.18 \\
\hline & & 10 & .134 & 1.01 \\
\hline & & 10 & .121 & .92 \\
\hline \multirow[t]{2}{*}{$1,086.30$} & 14.29 & 310 & .0945 & 1.35 \\
\hline & & 83 & .087 & 1.24 \\
\hline \multicolumn{5}{|c|}{ Negative. } \\
\hline \multicolumn{2}{|c|}{ Pressure. } & \multirow{2}{*}{$V$} & \multirow{2}{*}{$k$} & \multirow{2}{*}{$p k$} \\
\hline $\mathrm{Cm}$. of $\mathrm{Hg}$. & Atmos. & & & \\
\hline \multirow[t]{3}{*}{379.85} & 5.00 & 485 & .330 & 1.65 \\
\hline & & 9.5 & .300 & 1.50 \\
\hline & & 9 & .293 & 1.46 \\
\hline \multirow[t]{5}{*}{558.14} & 7.34 & 408 & .253 & 1.86 \\
\hline & & 408 & .250 & 1.84 \\
\hline & & 82 & .241 & 1.77 \\
\hline & & 82 & .246 & 1.73 \\
\hline & & 10 & .223 & 1.64 \\
\hline \multirow[t]{2}{*}{$1,033.60$} & 13.60 & 755 & .130 & 1.76 \\
\hline & & 10 & .118 & 1.60 \\
\hline \multirow[t]{2}{*}{$1,550.40$} & 20.40 & 800 & .092 & 1.87 \\
\hline & & 29.5 & .0895 & 1.78 \\
\hline
\end{tabular}

This effect may possibly in part be due to the lines of force, with higher potentials, extending further into the chamber $G S$, but the larger effect 
with the positive ion appears to be due to an actual change in the value of $k$.

A few measurements were also made at atmospheric pressure by placing the polonium on $G$ facing $P$. The same effect was noticed but the results obtained in this set of measurements were not so reliable as the contacts on the commutator were not so definite at the increased speed of running.

\section{Summary of Results.}

I. The mobility of the positive ion made by the alpha rays from polonium in air has been shown to vary inversely with the pressure up to 100 atmospheres.

II. At high pressures the mobility of the negative ion made in air by the alpha rays from polonium does not appear to vary inversely with the pressure but seems to decrease less rapidly with the pressure than it should if it followed this law.

III. From a series of observations with Frank and Pohl's method it has been shown that the mobility of the negative ion made in air by the alpha rays from polonium is greater with high voltages applied to the antechamber of the ionization vessel than with low ones.

In conclusion I wish to express my thanks to Professor McLennan who suggested the problem and aided in every possible way throughout the progress of the experiment.

The Physical Laboratory, UNIVERSITY OF TORONTO, October 20, rgro. 